Research Article

\title{
Equivalent Locally Martingale Measure for the Deflator Process on Ordered Banach Algebra
}

\author{
Boushra Y. Hussein (iD) \\ Department of Mathematics, College of Education, University of Al-Qadisiyah, Al Diwaniyah, Iraq \\ Correspondence should be addressed to Boushra Y.Hussein; boushra.alshebani@qu.edu.iq
}

Received 13 March 2020; Revised 22 April 2020; Accepted 14 May 2020; Published 9 June 2020

Academic Editor: Antonio Di Crescenzo

Copyright ( $\odot 2020$ Boushra Y. Hussein. This is an open access article distributed under the Creative Commons Attribution License, which permits unrestricted use, distribution, and reproduction in any medium, provided the original work is properly cited.

\begin{abstract}
This paper aims at determining the measure of $Q$ under necessary and sufficient conditions. The measure is an equivalent measure for identifying the given $P$ such that the process with respect to $P$ is the deflator locally martingale. The martingale and locally martingale measures will coincide for the deflator process discrete time. We define $s$-viable, $s$-price system, and no locally free lunch in ordered Banach algebra and identify that the s-price system $(C, \pi)$ is $s$-viable if and only a character functional $\left.\psi\right|_{C} \leq \pi$ exists. We further demonstrate that no locally free lunch is a necessary and sufficient condition for the equivalent martingale measure $Q$ to exist for the deflator process and the subcharacter $\phi \in \Gamma$ such that $\left.\varphi\right|_{C}=\pi$. This paper proves the existence of more than one condition and that all conditions are equivalent.
\end{abstract}

\section{Introduction}

In this paper, we combine certain concepts in the functional analysis with other concepts in financial mathematics to generate new results. These results are crucial to the improvement of efficient markets and the stock market. Czkwanianc and Pazkiewicz [1] highlighted the martingale measure for the stochastic process with discrete time; Harrison and Kreps [2] investigated the fundamental theorem and confirmed that the equivalent martingale measure is not sufficient to no-arbitrage alone for the stochastic process.

Many researchers have discussed the different methods for stopping time. Delbaen [3] introduced the martingale measure in continuous time and bounded. Kerps [4] presented a new definition of the no-arbitrage concept with certain properties. Dalang et al. [5] discussed the relationship between the equivalent martingale measure and no-arbitrage in stochastic securities. Harrison and Kreps [6] introduced the martingales and stochastic inferals in the theory of continues trading. Back and Pliska [7] identified the fundamental theorem of asset pricing with infinite state space. Schachermayer [8] employed the concept of no-arbitrage and built upon the work of David Kreps to identify the relation between equivalent measure and no-free lunch. Shachermayer [9] validated the fundamental theorem in Hilbert space. Delbaen and Shachermayer [10, 11] developed the fundamental theorem of asset pricing for unbounded stochastic process.

Clark [12] created an equitable relationship between the martingale measure and the extension property while Schachermayer [13] determined the equivalences between the martingale measure in discrete time and arbitrage. Kabanov and Stricker [14] employed the equivalent martingale measure with bounded densities. Kabanov and Safarian [15] introduced the new properties for the function markets with transaction costs. Chen [16] introduced the viable costs and equilibrium price in frictional securities markets. Gaussel [17] identified the martingale property of prices when arbitrage opportunities can be found. Kabanov [18] built upon the studies of earlier researchers by introducing the concept of the locally martingale measure. Hussein $[19,20]$ found the equivalent (super/sub) martingale measure and discussed the equivalent martingale in $L^{P}$-space. Hussein and Fahim [21] proved new properties of the character in ordered Banach algebra. Prkaj and Ruf [22] determined the locally martingale measure in discrete time.

In this paper, we introduce the deflator process and determine the necessary and sufficient conditions of 
equivalent locally martingale for deflator process using ordered Banach algebra and other analytic concepts, such as algebra cone and character. We define certain concepts such as $s$-viable, $s$-price system, and $s$-no free lunch. We demonstrate the necessary and sufficient condition for the existence of the equivalent locally martingale measure. Different solutions have been introduced in relation to the topological conditions of arbitrage. Generally, some results validate the relation of no-arbitrage conditions to the existence of an equivalent locally martingale measure for deflator process.

The triple $(\Omega, F, P)$ is called the probability space, where $\Omega$ is a nonempty, set, $F$ is a $\sigma$-field on $\Omega$, and $P$ is a probability measure. The process $S$, sometimes denoted as $\left(S_{t}\right)_{t \in I}$, is the process adapted to filter $\left\{F_{t}\right\}_{t \in I}$. The probability measure $Q$ defined on $F$ is equivalent to $P$ if $Q$ and $P$ contain the same null sets. The equivalent probability measure $Q$ is the equivalent martingale measure to $\left(S_{t}\right)_{t \in I}$ if $\left(S_{t}\right)_{t \in I}$ is martingale with respect to $Q$. Thus, if $S$ is integral with respect to $Q$ and for all $t \in I, E\left(\left(S_{t}-S_{t-1}\right) / F\right)=0$. A probability measure $Q$ is an equivalent local martingale measure for $S$ if $Q$ is equivalent to $P$ and $S$ is $Q$-local martingale.

This paper is divided into four sections. Section 1 introduces the research. Section 2 explains the concepts and definitions required in the main subject as locally martingale, ordered Banach algebra, trading strategy, deflator process, subcharacter, s-price system, s-viable, and, s-no arbitrage. The classes of martingale and locally martingale measures coinciding with the discrete-time filtrated probability measure are discussed, and the existence of a one-to-one correspondence between equivalent martingale measure $Q$ for the deflator process and the subcharacter $\varphi \in \Gamma$ such that $\left.\varphi\right|_{C}=\pi$ and some properties are proposed. In Section 3, the relationship between the equivalent locally martingale measure and the s-no-arbitrage is determined and the s-price system $(C, \pi)$ is confirmed to be no-arbitrage if and only if a deflator process exists. An equivalent locally martingale measure exists if and only if a uniformly integrable deflator process exists. We prove that if $S$ is a $\mathfrak{R}^{d+1}$-valued semimartingale with nonnegative components defined on the filtered probability space $\left(\Omega, F,\left\{F_{t}\right\}_{t \in I}, \mathrm{P}\right)$, an equivalent local martingale measure for deflator process also exists. Section 4 shows the relation between the deflator process and s-no-arbitrage, and we prove the uniformly integrable deflator process is necessary and sufficient condition to existence the equivalent locally martingale measure. Section 5 discusses the important conclusions of the research.

\section{Preliminary}

Consider a filtered probability space $\left(\Omega, F,\left\{F_{t}\right\}_{t \in I}, P\right)$ where $I \subset[0,1]$ with $0, T \in I$, for a fixed $T<\infty$. Let $F_{0}=\{F, \Omega\}$, $F_{\mathrm{T}}=F, S=(S(t))_{t \in I}$ be a $\Re^{d+1}$-valued stochastic process with components $S_{i}(t)$ for $i=0,1, \ldots, d$ and satisfy the following properties:

(1) $S_{i} i s$ an adapted to $\left\{F_{t}\right\}_{t \in I}$ for all $i=0,1, \ldots, d$

(2) $E\left(\left(S_{i}\right)^{2}(t)\right)<\infty$ for $t \in I$ and for all $i=0,1, \ldots, d$

(3) $S_{0}(t, \omega)=1$ for all $t \in I$ and for all $\omega \in \Omega$.
Definition 1 (see [22]). Let $(\Omega, F, P)$ be a complete probability space and $\left\{F_{t}: t \geq o\right\}$ be a right continuous filtration. A right continuous adapted process $\left\{S_{t},\left\{F_{t}: t \geq 0\right\}\right.$ is locally martingale if there exists a sequence $\left\{\tau_{n}: n \geq 1\right\}$ of stopping time of filtration satisfying the following:

(1) $\tau_{n} \leq \tau_{n+1}$

(2) $P\left[\tau_{n} \leq n\right\}=1$

(3) $P\left[\lim _{n} \tau_{n}=\infty\right\}=1$

(4) If $\tau_{t}^{n}=T_{n \wedge t}, S_{t}^{n}=S \circ \tau_{t}^{n}$

Then, for each $n,\left\{S_{t}^{n}, F_{t}, t \geq 0\right\}$ is a uniformly integrable martingale. This concept plays a key role in the analysis. A positive local martingale $\left\{S_{t}^{n}, F_{t}, t \geq 0\right\}$ is super martingale, that is, let $A \in F_{s}, 0 \leq s \leq t$. Now, the set $\left\{X \circ \tau_{t}^{n}, t \geq\right.$ 0 \}is uniformly integrable.

For all $\alpha \geq 0, \quad\left\{w: \lim _{n} \tau_{n}=\infty\right\}=\left\{\cup_{n \geq 1} \lim _{n} \tau_{n} \geq \alpha\right\}=$ $\cup_{n \geq 1} \mathbf{B}_{n, \alpha}$ differs from $\Omega$ by a $P$-null set and thus $\mathbf{B}_{n, \alpha} \in F_{\alpha}$ for all $n \geq 1$ and $\mathbf{B}_{n, \alpha} \subset \mathbf{B}_{n+1, \alpha} \uparrow \Omega$ (almost everywhere).

Hence,

$$
\int_{A} X_{t} \mathrm{~d} P=\lim _{n} \int_{A \cap \mathbf{B}_{n, t}} X_{t} \mathrm{~d} P=\lim _{n} \int_{A \cap \mathbf{B}_{n, t}} X_{t}{ }^{\circ} \tau_{\mathrm{t}}^{\mathrm{n}} \mathrm{dP},
$$

because $X \circ \tau_{t}^{n}=X_{t}$ on

$$
\begin{aligned}
\mathbf{B}_{n, t} & \leq \lim _{n} \int_{A \cap \mathbf{B}_{n, s}} X_{t}^{\circ} \tau_{\mathrm{t}}^{\mathrm{n}} \mathrm{dP}\left(\mathbf{B}_{\mathrm{n}, \mathrm{t}} \subset \mathbf{B}_{\mathrm{n}, \mathrm{s}} \text { for } \mathrm{s}<\mathrm{t} \text { and } \mathrm{X}_{\mathrm{t}} \geq 0\right) \\
& =\lim _{n} \int_{A \cap \mathbf{B}_{n, s}} X_{t}{ }^{\circ} \tau^{\mathrm{n}} \mathrm{dP}, \quad \text { by martingale property of }\left\{\mathrm{X} \circ \tau_{\mathrm{s}}^{\mathrm{n}}, \mathrm{s} \geq 0\right\} \\
& =\int_{A \cap \mathbf{B}_{n, t}} X_{t} \mathrm{~d} P=\int_{A} X_{t} \mathrm{~d} P, \quad \text { then } E\left(X_{t} \mid F_{s}\right) \leq X_{s} .
\end{aligned}
$$

Definition 2 (see [23]). An algebra $A$ is a linear space whose vectors can be multiplied in such a way that

(1) $(x \cdot y) \cdot z=x \cdot(y \cdot z)$

(2) $(x+y) z=x z+y z$ and $z(x+y)=z x+z y$

(3) $\alpha(x y)=(\alpha x) y=x(\alpha y)$ for all scalar $\alpha$

We speak of a complex or a real algebra according as the scalars are complex or real numbers. A commutative algebra is an algebra where multiplication satisfies the following condition:

$$
x y=y x, \quad \text { for all } x, y \in A .
$$

An algebra with identity is an algebra with the following property: there exists a nonzero element in the algebra, denoted by $e$ and called the multiplicative identity element (or simply the identity), such that $e \cdot x=x \cdot e=x$, for all $x$.

Definition 3 (see [23]). If $A$ is a normed linear space and also an algebra over $\mathbb{C}($ or $\mathbb{R})$, and if $x \cdot y \leq x \cdot y$, for all $x, y \in A$, then $A$ is called a complex (or real) normed algebra. If a normed algebra has the multiplicative identity, then we will postulate that $\|e\|=1$.

A complex (real) Banach apace which is also a normed algebra is called Banach algebra. 
Definition 4 (see [21]). Let A be a real Banach algebra with identity 1 and $C$ nonempty subset of $A$. We call $C$ a cone if the following hold:

(1) $a+b \in C$ for all $a, b \in \mathrm{C}$

(2) $\lambda a \in C$ for all $a \in C$ and $\lambda \geq 0$

If $C \cap-C=\{0\}$, then $C$ will be called a proper cone. Any cone $\mathrm{C}$ on $\mathrm{A}$ induced an ordering $\geq$ on $A$ as follows: $b \geqslant a$ if and only if $b-a \in C$ for all $a, b \in C$.

We say that $C$ is algebra cone if

(1) $a \cdot b \in C$ for all $a, b \in C$

(2) $e \in C$

Definition 5 (Ordered Banach algebras, see [21]). A Banach algebra $A$ with unite $A$ is called ordered Banach algebra, which is denoted by (OBA) when $A$ is ordered by a relation $\geqslant$ such that for every $a, b \in A$ and $\beta \geqslant 0$,

(1) $a, b \geqslant 0 \Rightarrow a+b \geqslant 0$

(2) $a \geqslant 0, \beta \geqslant 0 \Rightarrow \lambda a \geqslant 0$

(3) $a, b \geqslant 0 \Rightarrow a b \geqslant 0$

(4) $e \geqslant 0$

So, if $A$ is ordered by an algebra cone $C$, we will obtain $(A, C)$ as an ordered Banach algebra.

If $A$ is an $(O B A)$ and $C$ is an algebra cone, $C$ is called normal if there is $\beta>0$, for any $a, b \in A, 0 \leq a \leq b<\Rightarrow$ $\|a\| \leq \beta\|b\|$.

Theorem 1 (see [24]). Let $M$ and $N$ locally martingales and $S$ and $T$ be stopping times. Then,

(1) The sum $M+N$ is also local martingale

(2) If $T$ reduces $M$ and $S \subseteq T$, then $S$ reduces $M$

(3) If both $S$ and $T$ reduce, then $S \cup T$ also reduces

(4) The processes $M^{T}$ and $M_{\{T>0\}}^{T}$ are local martingale

\section{Existence of the Equivalent Martingale Measure for the Deflator Process in Ordered Banach Algebra}

We start the section by definition of trading strategy as follows:

Definition 6. A trading strategy $H$ is an $R^{d+1}$-valued vector stochastic process, $H=\left(\varphi(t)^{0}, \ldots, \varphi(t)^{d+1}\right)_{t=1}^{T}$, such that $\varphi(t)$ is a $F_{t-1}$ - measurable function for all $t \geq 1$, integrable with respect to semimartingale $M=(S(t))_{t=1}^{T}$.

Let $V_{t}(H)=H \cdot M=\varphi_{t}^{0} S_{t}^{0}+\varphi^{t}, S^{t}=\varphi_{t}^{0} S_{t}^{0}+\sum_{j=1}^{m} \varphi_{t}^{j} S_{t}^{j}$, where $H=\left(\varphi(t)^{0}, \ldots, \varphi(t)^{d+1}\right)$.

The process $V_{t}(H)$ is called value process of $H$.

Definition 7. Let $S^{i}, i=0,1, \ldots, m$, be a strictly positive semimartingale; we set $S=\left(S^{1}, S^{2}, \ldots, S^{m}\right)$ and $(X(t))_{t=0}^{T}$ as a sequence of random variables, which is strictly positive for $t \in\{0,1, \ldots, T\}$. We call $\left((X(t))_{t=0}^{T}\right)^{-1}$ a deflator random variable, and a measurable function $\widetilde{S}=\left(\left(S^{1} /(X(t))\right)\right.$, $\left.\left(S^{2} /(X(t))\right), \ldots,\left(S^{m} /(X(t))\right)\right)$ is called deflator process.

Definition 8. A functional $\pi$ on ordered Banach algebra is a called a subcharacter if $\pi$ is a sublinear functional on $C$ and $\pi(x \cdot y)=\pi(x) \cdot \pi(y)$. The set of all subcharacters is denoted by $\Gamma$.

Definition 9. The s-price system is a pair $(C, \pi)$ where $C$ is an algebra cone of $A$ and $\pi$ is a subcharacter.

Definition 10. The s-price system $(C, \pi)$ is s-viable if $\pi(x) \leq 0$ and $x \pm y$ for all $y \in C$ such that $\pi(y) \leq 0$, in a special case if $e \geq x$ with $\pi(x) \leq 0$.

Definition 11. Let $(A, C)$ be (OBA) with the algebra cone $C=A_{+} \backslash\{0\}, K_{0} \subseteq X$, and $K=\operatorname{span}\left(K_{0}\right)$. Topology $\tau$ is weak topology, $\pi: C \longrightarrow \mathbb{R}$ is a strictly positive linear functional, and $(C, \pi)$ is s-no-arbitrage; if $x \in K \cap C$, then $\pi(x)>0$.

Theorem 2. The classes of the martingale and the locally martingale coincide on the discrete-time filtrated probability measure.

Proof. Take $M=\left(M^{i}\right)_{i \leq n}$ as a $A^{n}$-valued local martingale and $H=\left(H^{i}\right)_{i \leq n}$ as a $A^{n}$-valued predictable process. We define

$$
S=H \cdot M=\left\langle\varphi^{t}, S^{t}\right\rangle=\sum_{i=1}^{n} H^{i} M^{i}
$$

If $H^{i}$ is bounded and $M^{i}$ is martingale, then $S$ is martingale.

Otherwise, let $\left\{\tau_{n}\right\}$ be a sequence of stopping times increasing as to $\infty$, such that each process $M^{\tau_{n}}$ is a martingale and the process $S^{\tau_{n}}$ is martingale.

Let $S$ be martingale; that is, $S_{n}^{\tau_{n}}$ with the sequence $\tau_{n}$ each is integrable and $E\left[\left|S_{n+1}\right| \mid F_{n}\right]=E\left[S_{n+1}^{\tau_{n}} \mid F_{n}\right]$ on the $F_{n}$-measurable set $\left\{\tau_{n}>n\right\}$.

$E\left[\left|S_{n+1}\right| \mid F_{n}\right]=E\left[S_{n+1}^{\tau_{n}} \mid F_{n}\right]=S_{n}^{\tau_{n}}=S_{n}$. Take $u_{n}=\sum_{k \geq 0}$ $(1 /(k+1)) \Delta S_{n} I_{\left\{w: E\left[\Delta S_{n} \| F_{n-1}\right](w) \in[k, k+1)\right\}}$ as $F_{n}$-measurable integrable and $\left[u_{n} \mid F_{n}\right]=0$. Then, $M_{n}=\sum_{i=1}^{n} u_{i}$ is a martingale, and (4) holds.

Theorem 3. Let $A$ be ordered Banach algebra with algebra cone $(A, C)$. The s-price system $(C, \pi)$ is s-viable if and only if a character functional $\left.\psi\right|_{C} \leq \pi$ exists.

Proof. Suppose there exists a character functional $\left.\psi\right|_{C} \leq \pi$; let $h \in C$ with $\pi(h) \leq 0$. Because $\psi(h) \leq \pi(h)$ for all $h \in H$, then $\psi(h) \leq 0=\psi(0)$. That is, $\psi(0) \geq \psi(h)$. Hence, $h \geq e$; that is, $(C, \pi)$ is s-viable.

Conversely, suppose that s-price system is s-viable.

Define $\quad M=\{h \in A: h>e\}, N=\{h \in C: \pi(h) \leq 0\}$. If $h \in M \cap N$, then $h>e$ and $\pi(h) \leq 0$; this contradicts that $(C, \pi)$ is s-viable. Then, $M$ and $N$ are disjoint.

Because $C$ is cone, then $C$ is a convex set. To prove $N$ is cone, let $h_{1}, h_{2} \in C$ and $0 \leq \lambda, 0 \leq \beta, \pi\left(h_{1}\right) \leq 0$, and $\pi\left(h_{2}\right) \leq 0$; 
that is, $\lambda \pi\left(h_{1}\right) \leq 0$ and $\beta \pi\left(h_{2}\right) \leq 0$. Then, $\lambda \pi\left(h_{1}\right)+(\beta) \pi\left(h_{2}\right) \leq 0$; that is, $\pi\left(\lambda h_{1}+\beta h_{2}\right) \leq 0$. $\pi$ is character functional; then $\lambda h_{1}+\beta h_{2} \in C$ and $N$ is cone.

Thus, $M$ and $N$ are disjointed, nonempty convex sets in (OBA). Using the separation theorem, there exists a nontrivial continuous linear functional $\psi$ on $A$ such that $\psi(h)>0$ for all $h \in$ Mand $\psi(h) \leq 0$ for all $h \in N$.

If $\psi(h \cdot g) \neq \psi(h) \cdot \psi(g)$, then $\psi(h \cdot g)>\psi(h) \cdot \psi(g)$ or $\psi(h \cdot g)<\psi(h) \cdot \psi(g)$.

By taking $h \in M$ and $g \in N$, then $\psi(h) . \psi(g) \leq 0$; that is, $\psi(h \cdot g) \leq 0$, to prove $\psi\left(h_{0}\right)>0 . \psi$ is nontrivial, and thus, $x_{0} \in A$ exists, such that $\psi\left(h_{0}\right)>0$.

$M$ is cone and we have $h_{0}-\lambda x_{0}>\theta$ for $\lambda>o$ and $h_{0} \in H$. Therefore, $\psi\left(h_{0}-\lambda x_{0}\right) \geq 0$, and by linearity of $\psi$, we have $\psi\left(m_{0}\right)-\lambda \psi\left(x_{0}\right) \geq 0$, and when $\psi\left(m_{0}\right) \geq \lambda \psi\left(x_{0}\right)>0$, we get $\psi\left(h_{0}\right)>0$. As $\pi\left(h_{0}\right)>0$ and $\psi\left(h_{0}\right)>0, \psi$ can be normalized so that $\psi\left(h_{0}\right)=\pi\left(h_{0}\right)$.

To prove $\left.\psi\right|_{M} \leq \pi$, let $h \in M$. If $\pi(h) \leq 0$ and $\lambda>0$ such that $\pi(h)+\lambda \pi\left(h_{0}\right)=0, \quad \pi\left((1 /(\lambda+1))+(\lambda /(\lambda+1)) h_{0}\right) \leq$ $(1 /(\lambda+1)) \pi(h)+(\lambda /(\lambda+1)) \pi\left(h_{0}\right) \leq(1 /(\lambda+1))[\pi(h)+\lambda \pi$ $\left.\left(h_{0}\right)\right]=0$ for $h, h_{0} \in C$, and $C$ is cone, then we have $(1 /(\lambda+1)) h+(\lambda /(\lambda+1)) h_{0} \in C$. Therefore, $\psi((1 /(\lambda+$ $\left.1)) h+(\lambda /(\lambda+1)) h_{0}\right) \leq 0, \quad((1 /(\lambda+1)) \psi(h)+(\lambda /(\lambda+1)) \psi$ $\left.\left(h_{0}\right)\right) \leq 0$; that is, $\psi(h) \leq \lambda \psi\left(h_{0}\right)=-\lambda \pi\left(h_{0}\right)=\pi(h)$.

Definition 12. An s-price is said to be s-no locally free lunch if a net $\left\{\left(m_{\alpha}, x_{\alpha}\right): \alpha \in \Lambda\right\} \subseteq C \times A$ and $x \in X_{+}$exist, such that

(1) $m_{\alpha} \geq x_{\alpha} \forall \alpha \in \Lambda$.

(2) $x_{\alpha} \stackrel{\tau}{\longrightarrow} x$, and $m_{\alpha} \longrightarrow m$ such that $\pi(m) \leq \delta \forall \alpha \in \Lambda$. For $\delta \in R, m_{\alpha} \in C$.

Proposition 1. The following conditions are equivalent:

(1) The s-price system $(C, \pi)$ is no locally free lunch

(2) $\widetilde{C} \cap X_{+} \neq \phi$, where $\widetilde{C}$ is the set of all limit points of convergence net in $C$

Proof. Suppose that $(C, \pi)$ admits an s-locally free lunch. Then, a net $\left\{\left(m_{\alpha}, x_{\alpha}\right): \alpha \in \Lambda\right\} \subseteq C \times X$ and $x \in X_{+}$exists such that

(1) $m_{\alpha} \geq x_{\alpha} \forall \alpha \in \Lambda$

(2) $x_{\alpha} \stackrel{\tau}{\longrightarrow} x$ and $m_{\alpha} \longrightarrow m$ such that $\pi(m) \leq \delta$ for all $\alpha \in \Lambda$ and for $\delta \in \mathbb{R}$

Then, $m_{\alpha} \in C$ because $m_{\alpha} \geq x_{\alpha}$, and $x_{\alpha}-m_{\alpha} \in C$ for all $\alpha \in \Lambda$.

$x_{\alpha} \stackrel{\tau}{\longrightarrow} x$ and $m_{\alpha} \longrightarrow m$, and then, $x-m \in C$; we have $x \geqslant m$, that is, $m \in \widetilde{C}$.

$x \in X_{+}$and $x \geqslant e$ and $x \geqslant m$; we obtain $m \geqslant e$, which implies that $m \in m \in \widetilde{C} \wedge m \in X_{+}$.

Hence, $m \in \widetilde{C} \cap X_{+}$; that is, $\widetilde{C} \cap X_{+} \neq \phi$.

Conversely, suppose $\widetilde{C} \cap X_{+} \neq \phi$. Then, $y \in \widetilde{C} \cap X_{+}$exists, that is, $y \in \widetilde{C}$ and $y \in X_{+}$.

$x_{\alpha} \in C$ such that $x_{\alpha} \stackrel{\tau}{\longrightarrow} y$ and $m_{\alpha} \in C$ such that $x_{\alpha} \geq m_{\alpha}$ for all $\alpha \in \Lambda$. $m_{\alpha} \in C$ and $\pi(m) \leq \delta$, for all $\alpha \in \Lambda$.
Theorem 4. If no locally free lunch exists, then a one-to- one correspondence between the equivalent martingale measure $Q$ for the deflator process and the subcharacter $\varphi \in \Gamma$ exists such that $\left.\varphi\right|_{C}=\pi$. This correspondence is given by $Q(A)=\varphi\left(I_{A}\right)$ and $\varphi(x)=E_{Q}(x)$.

Proof. Let $Q$ be an equivalent measure; set $\rho=(\mathrm{d} Q / \mathrm{d} P)$.

Define $\psi: X \longrightarrow R$ by $\psi(x)=E_{Q}(x)$ for all $x \in X$. Then, $\psi(x)=E(\rho x)$ and $\rho \in L^{2} ; \psi$ is linear functional and continuous:

$$
\psi(x \cdot y)=E_{Q}(x \cdot y)=E_{Q}(x) \cdot E_{Q}(y)=\psi(x) \cdot \psi(y) .
$$

Then, $\psi$ is character. $Q \sim P$ and $\rho$ are strictly positive, and thus, $\psi$ is strictly positive.

Take $\varphi=\psi$, that is, $\varphi \in \Gamma$; this remains to show that $\left.\psi\right|_{M}=\pi$.

For $n=1,2, \ldots, k$, we have $E_{Q}\left(\left\langle\vartheta\left(t_{n}\right), \widetilde{S}\left(t_{n}\right)\right\rangle /\right.$ $\left.F_{t_{n-1}}\right)=E_{Q}\left[\left\langle\vartheta\left(t_{n-1}\right), \widetilde{S}\left(t_{n}\right)\right\rangle / F_{t_{n-1}}\right]$ :

$$
\begin{aligned}
\left\langle\vartheta\left(t_{n}\right), \widetilde{S}\left(t_{n}\right)\right\rangle & =\left\langle\vartheta\left(t_{n-1}\right), \widetilde{S}\left(t_{n}\right)\right\rangle, \\
E_{Q}\left(\frac{\left\langle\vartheta\left(t_{n}\right), \widetilde{S}\left(t_{n}\right)\right\rangle}{F_{t_{n-1}}}\right) & =\frac{\left\langle\vartheta\left(t_{n-1}\right), E_{Q}\left(\widetilde{S}\left(t_{n}\right)\right)\right\rangle}{F_{t_{n-1}}} \\
& =\left\langle\vartheta\left(t_{n-1}\right), \widetilde{S}\left(t_{n-1}\right)\right\rangle .
\end{aligned}
$$

Because $\widetilde{S}$ is a martingale with respect to $Q$, this equality yields $E_{Q}(\langle\vartheta(T), \widetilde{S}(T)\rangle)=\langle\vartheta(0), \widetilde{S}(0)\rangle . V^{\vartheta}(T)=h$; that is, $\langle\vartheta(T), \widetilde{S}(T)\rangle=h$; then, $\quad E_{Q}(h)=\langle\vartheta(0), \widetilde{S}(0)\rangle, \quad \pi(h)=$ $V^{\vartheta}(0)=\langle\vartheta(0), \widetilde{S}(0)\rangle$, and thus $E_{Q}(h)=\pi(h), \psi(h)=\pi(h)$ for all $m \in C$.

Conversely, let $\varphi \in \Gamma$ such that $\left.\varphi\right|_{M}=\pi$. Define $Q$ : $F \longrightarrow \mathfrak{R}$ by $Q(X)=\varphi\left(I_{X}\right)$ for all $X \in F$.

If $P(X)=0$, that is, $I_{X}=0$, then $Q(X)=\varphi(0)=0$.

If $P(X)>0$, then $I_{X} \in H_{+}$. Therefore, $\phi\left(I_{X}\right)>0$, that is, $Q(X)>0$.

To prove that $Q$ is measure $Q(\phi)=\varphi\left(I_{\varnothing}\right)=0$ and $Q\left(\cup_{i=1}^{\infty} E_{i}\right)$, where $E_{i} \in F=\varphi\left(I_{\cup_{i=1}^{\infty} E_{i}}\right)=\varphi\left(\sum_{i=1}^{\infty} I_{E_{i}}-\sum_{i \neq j=1}^{\infty}\right.$ $\left.I_{E_{i} \cap E_{j}}\right)=\varphi\left(\lambda \sum_{i=1}^{\infty} I_{E_{i}}+(1-\lambda) \quad \sum_{i \neq=1}^{\infty} I_{E_{j}}\right) E_{i} \cap E_{j}=\varphi \phi \leq$ $\sum_{i=1}^{\infty} \varphi\left(I_{E_{i}}\right)=\sum_{i=1}^{\infty} Q\left(I_{E_{i}}\right)$, then $Q$ is measure. Also, to prove the measure $Q$ is equivalent to measure $P$.

If $P(X)>0$, then

$I_{X}$ is positive linear functional, $\varphi\left(I_{X}\right)>0$; that is, $Q(X)>0$.

$\varphi$ is continuous, so $Q$ is a $\sigma$-additive measure, and $\mathrm{d} Q$ / $\mathrm{d} P=\rho$ is square-integrable.

$I_{\Omega}=1$ if $x \in \Omega$ and 0 if $x \notin \Omega$; then, $\varphi\left(I_{x}\right)=1$; that is, $Q(\Omega)=1$. Thus, $Q$ is probability measure equivalent to $P$.

Proposition 2. A trading strategy $H$ is self-financing with respect to the deflator process if and only if $H$ is self-financing with respect to $S(t)$. 
Proof

$$
\begin{aligned}
V_{t}(H) & =V_{t+1}(H), \\
\varphi_{t}^{0} S_{t}^{0}+\left\langle\varphi^{t}, S^{t}\right\rangle & =\varphi_{t+1}^{0} S_{t+1}^{0}+\left\langle\varphi^{t+1}, S^{t+1}\right\rangle, \\
\varphi_{t}^{0} S_{t}^{0}+\sum_{j=1}^{m} \varphi_{t}^{j} S_{t}^{j} & =\varphi_{t+1}^{0} S_{t+1}^{0}+\sum_{j=1}^{m} \varphi_{t+1}^{j} S_{t+1}^{j} .
\end{aligned}
$$

For any $(X(t))_{t=0}^{T}$, for all $t \in\{0,1, \ldots, T\}$, a sequence of strictly positive random variables exists as follows:

$$
\begin{gathered}
\varphi_{t}^{0}(X(t))^{-1} S_{t}^{0}+\sum_{j=1}^{m} \varphi_{t}^{j}(X(t))^{-1} S_{t}^{j} \\
=\varphi_{t+1}^{0}(X(t))^{-1} S_{t+1}^{0}+\sum_{j=1}^{m} \varphi_{t+1}^{j}(X(t))^{-1} S_{t+1}^{j} \\
\varphi_{t}^{0} \widetilde{S}_{t}^{0}+\sum_{j=1}^{m} \varphi_{t}^{j} \widetilde{S}_{t}^{j}=\varphi_{t+1}^{0} \widetilde{S}_{t+1}^{0}+\sum_{j=1}^{m} \varphi_{t+1}^{j} \widetilde{S}_{t+1}^{0} .
\end{gathered}
$$

Then, $H$ is self-financing with respect to the deflator process $\widetilde{S}_{t}$.

\section{Relation between Equivalent Locally Martingale Measure and S-No-Arbitrage}

In this section, we introduce important results related to the main subject.

Proposition 3. Let $(\Omega, F, P)$ be a probability space. Then, $(C, \pi)$ is s-no-arbitrage if and only if a deflator process exists.

Proof. Suppose $(C, \pi)$ is no-arbitrage. If $\widetilde{S} \in K \cap C$, that is, $\widetilde{S} \succ 0$.

Also, $\quad S_{0}=1$. For any process $X \in M$, the deflator process $\widetilde{S}(t)=(S(t) / X(t))$; then, $\pi(\widetilde{S})=E\left[\widetilde{S}_{n}\right]$.

Conversely, suppose a deflator process exists. This is equivalent to $S_{n} \in K$, that is, $S_{0}>0 . X_{0}=1$ and $\widetilde{S}_{0}=\left(S_{0} / X_{0}\right)=E\left[S_{n} / X_{n}\right]=E\left[\widetilde{S}_{n}\right]$. Then, $\pi\left(\widetilde{S}_{n}\right)=\widetilde{S}_{0}>0$.

Theorem 5. Let $(\Omega, F, P)$ be a probability space. Then, an equivalent locally martingale exists if and only if a uniformly integrable deflator process exists.

Proof. A measure $Q$ equivalent to $P$ with Radon-Nikodom derivative $\mathrm{d} Q_{t} / \mathrm{d} P_{t}=Z_{t}$ is strictly positive and $E\left[Z_{t}\right]=1$, for a given sequence $\left\{\tau_{n}\right\}$ with finite stopping time $v F \in F_{v}$,

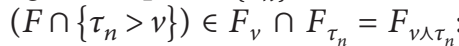

$$
\begin{aligned}
E & {\left[\chi_{F} E\left(\widetilde{S} \chi_{\left\{\tau_{n}>v\right\}} \mid F_{v \wedge \tau_{n}}\right)\right] } \\
& =\left[E\left(\widetilde{S} \mid F_{v \wedge \tau_{n}} \chi_{F \cap\left\{\tau_{n}>v\right\}}\right)\right] \\
& =E\left[E\left(\widetilde{S} \chi_{F \cap\left\{\tau_{n}>v\right\}} \mid F_{v \wedge \tau_{n}}\right)\right] E\left(\widetilde{S} \chi_{F \cap\left\{\tau_{n}>v\right\}}\right),
\end{aligned}
$$

where $E\left(\widetilde{S} \chi_{\left\{\tau_{n}>v\right\}} \mid F_{v \wedge \tau_{n}}\right)$ is $F_{v}$ - measurable, $E\left(\widetilde{S} \chi_{\left\{\tau_{n}>v\right\}} \mid\right.$ $\left.F_{v \wedge \tau_{n}}\right)=E\left(\widetilde{S} \chi_{\left\{\tau_{n}>v\right\}} \mid F_{v}\right)$, that is, $E\left(\widetilde{S} \mid F_{v \wedge \tau_{n}}\right) \chi_{\left\{\tau_{n}>v\right\}}=$ $E\left(\widetilde{S} \mid F_{\{v\}}\right) \chi_{\left\{\tau_{n}>v\right\}}$, and $Z \widetilde{S}$ is itself a local martingale.

Then, for any $S \in M, \pi(\widetilde{S})=E_{P}^{0}(S / X)=E_{Q}(\widetilde{S})$ and $\widetilde{S}_{i}=E_{P}^{i}\left[Z_{t}\right], \lim E\left[Z_{t} \widetilde{S}_{i}\right]=E\left[Z_{t}\right]=1$.
Then, $\widetilde{S}_{i}$ is uniformly integrable.

Conversely, if $\left\{\widetilde{S}_{i}\right\}$ is a uniformly integrable deflator process. Then, $\widetilde{S}_{i} \longrightarrow Z_{t} \in \ell^{1}(P)$ and $E\left[Z_{t}\right]=1$.

Choose $Q$ as a probability measure on the space $(\Omega, F)$ equivalent to $P$; then, with Radon-Nikodym theorem, it becomes $\left(\mathrm{d} Q_{t} / \mathrm{d} P_{t}\right)=Z_{t}$, so that $Q_{u}:=Q[\{u\}]=$ $Z_{t}(u) P[\{u\}]$ for $u \in A$.

For any $S \in M, E_{P}^{0}(S / X)=\pi(\widetilde{S})$, but $\pi(\widetilde{S})=E_{Q}(\widetilde{S})$. Then, $E_{P}^{0}(S / X)=E_{Q}(\widetilde{S})$ and the $P$-martingale property of $\tilde{S}$ is $Q$-martingale property of $S$.

Theorem 6. Let $S$ be a $\mathfrak{R}^{d+1}$-valued semimartingale with nonnegative components defined on the filtered probability space $\left(\Omega, F,\left\{F_{t}\right\}_{t \in I}, P\right)$. Then, equivalent local martingale measure for deflator process exists.

Proof. Let $\Theta$ be a set of all nonnegative processes, $D=\left\{\widetilde{X} \in \Theta: \widetilde{X}_{T}>0\right\}$ and $\preccurlyeq$ be a binary relation on $\Theta$ defined as

$$
\widetilde{X} \preccurlyeq \tilde{Y} \text { iff } \widetilde{X}_{0} \leq \widetilde{Y}_{0} \text { and } \tilde{X}-\tilde{Y} \text { is a supermartingale. }
$$

This order is not preferences ordered, if we have $A$ to the quotient space obtained from $\Theta$ by identifying processes whose difference is locally martingale null at 0 ; the pair $(A, \preccurlyeq)$ is an ordered linear space. Let $C$ be a set of all $\widetilde{X}$ in $A$ such that $\widetilde{X} \leq 1$. Then, the closure $\bar{C}=\{\widetilde{X} \in \Theta: E$ $[\tilde{X} \widetilde{Y}] \leqslant 1$ for all $\widetilde{Y} \in D\}$. Define $T: \quad \bar{C} \longrightarrow R$ as follows: $T(\tilde{X})=E[\widetilde{X}]$ for all $\tilde{X} \in \bar{C}$.

Then, $\bar{C} \cap A^{++}=\varnothing$, because for every $\tilde{X} \in \bar{C}$, we have $q \leq 0$ for some $q \in \bar{P}(\widetilde{X})$; let $\widetilde{X}_{\circ} \in A^{++}$. Then, $\widetilde{X} \notin \bar{C}$. But $C-$ $A^{+} \subseteq C$ implies $\overline{C-A^{+}} \subseteq \bar{C}$.Thus, $\widetilde{X} \notin \overline{C-A^{+}}$. So there exists an open, convex neighborhood of $\widetilde{X}$. Say $N(\widetilde{X})$, such that $N(\widetilde{X}) \cap\left(\overline{C-A^{+}}\right)=\varnothing . \quad$ In particular, $N(\widetilde{X}) \cap\left(C-A^{+}\right)=\varnothing$.

Let $C(\widetilde{X})$ denote the convex cone generated by $(N(\widetilde{X}))$ by $C(\widetilde{X})=\{\widetilde{Y} \in A: \widetilde{Y}=\lambda \widetilde{X}$ for some $\lambda>0$ and $\widetilde{X} \in N(\widetilde{X})\}$. Then, $C\left(\widetilde{X}_{0}\right) \cap\left(C-A^{+}\right)=\varnothing$, and by Hahn-Banach theorem, a continuous linear functional $P: A \longrightarrow R$ such that $P(\widetilde{X}) \geq 0$ for every $\widetilde{X} \in C(\widetilde{X})$ and $P(\widetilde{X}) \leq 0$ for every $\widetilde{X} \in C-A^{+}$.In particular, $P(\widetilde{X}) \leq 0$ for every $\widetilde{X} \in \bar{C}$. Hence, we obtain $\bar{P}: \bar{C} \longrightarrow R$ as a strictly positive character.

For every $\widetilde{X} \in C, P(\widetilde{X}) \leq 0$ implies $P(\widetilde{X}) \leq 0$ replacing $m$ with $-\tilde{X}$ in this implication.

We see that for every $\widetilde{X} \in C P(\widetilde{X}) \geq 0$ and $P(\widetilde{X}) \geq 0$, $P(\widetilde{X})=0$ implies $P(\widetilde{X})=0$ for every $\widetilde{X} \in C$, and there exists some $\lambda \in R$ such that $P(\widetilde{X})=\lambda P(\widetilde{X})$ for every $\tilde{X} \in C$, $\widetilde{X}_{0} \in C^{++}$, and $P\left(\widetilde{X}_{0}\right)>0 ; \bar{P}$ is continuous on C. Hence, $\bar{P}: \bar{C} \longrightarrow R$ is continuous, positive linear functional.

Finally, we show that $\bar{P}$ is strictly positive. First, assume that $\bar{P}$ is not strictly positive. Then, there exists $\widetilde{X} \in \bar{C}^{++}$such that $\bar{P}(\tilde{X})=0$. Let $\left\{\tilde{X}_{\alpha}: \alpha \in \Gamma\right\}$ be a net in $C$ such that $\widetilde{X}_{\alpha} \longrightarrow \widetilde{X} . \bar{P}\left(\widetilde{X}_{\alpha}\right) \longrightarrow \bar{P}(\widetilde{X})=0$. So, $P\left(\widetilde{X}_{\alpha}\right)>0$ for all $\alpha$ sufficiently large in the directed index set.

Define $\beta_{\alpha}=\left(P\left(\widetilde{X}_{\alpha}\right) / P\left(\widetilde{X}_{0}\right)\right)$ for every $\alpha \in \Gamma, \beta_{\alpha}>0$ for all $\alpha$ sufficiently large, and $\beta_{\alpha} \longrightarrow 0$.

Define $\widetilde{X}_{\alpha}^{*}=\widetilde{X}_{\alpha}-\beta_{\alpha} \widetilde{X}_{0}$ for every $y \in \Gamma$ and $\widetilde{X}_{\alpha}^{*} \in C$. 
Also, $P\left(\widetilde{X}_{\alpha}^{*}\right)=P\left(\widetilde{X}_{\alpha}\right)-\beta_{\alpha} P\left(\widetilde{X}_{0}\right)=0$ implies $\widetilde{X}_{0} \in \bar{C}$, and thus $\widetilde{X}_{0} \in \bar{C} \cap \bar{A}^{++}$is a contradiction. $\bar{P}$ is strictly positive. To prove any process under condition is locally martingale, let $M_{Q}$ be a set of all measures $Q$ equivalent to measure $P$, by taking $Y_{t}^{\mathrm{Q}}=E\left[(\mathrm{dQ} / \mathrm{d} P) \mid F_{t}\right] . A_{t}$ is a nondecreasing, adapted process such that $A_{0}=0$, and we assume $X_{0}=1$ in $[0, T]$ and find $H$ to be a predictable S-integrable process such that $X=1+\int_{0}^{t} H_{v} \mathrm{~d} S_{v}$. We set $\widetilde{Y}_{v}=\left(X_{s} /\left(X_{s}-A_{s}\right)\right) H_{v}$, so that $Y$ is an S-integrable predictable process:

$$
1+\int_{0}^{t} H_{v} \mathrm{~d} S_{v}= \begin{cases}X_{v}, & v \leq s, \\ X_{s}+\frac{X_{s}}{X_{s}-A_{s}}\left(X_{v}-X_{s}\right), & v>s .\end{cases}
$$

$$
X_{s}+\left(X_{s} /\left(X_{s}-A_{s}\right)\right)\left(X_{v}-A_{v}-\left(X_{s}-A_{s}\right)\right)=X_{s}\left(\left(X_{v}-A_{v}\right) /\right.
$$
$\left.\left(X_{s}-A_{v}\right)\right) \geq 0$; thus, this process is supermartingale:

$$
\begin{aligned}
\widetilde{Y}_{t}^{Q} X_{s} & =\widetilde{Y}_{s}\left(1+\int_{0}^{t} H_{v} \mathrm{~d} S_{v}\right) \geq E\left[\widetilde{Y}_{s}\left(1+\int_{0}^{t} H_{v} \mathrm{~d} S_{v}\right) \mid F_{t}\right] \\
& =\frac{X_{s}}{X_{s}-A_{s}} E\left[\widetilde{Y}_{s}\left(X_{t}-A_{s}\right) \mid F_{s}\right] \\
& \geq \frac{X_{s}}{X_{s}-A_{s}} E\left[\widetilde{Y}_{s}\left(X_{t}-A_{t}\right) \mid F_{t}\right] \\
& \geq \frac{X_{s}}{X_{s}-A_{s}} E\left[\widetilde{Y}_{s}\left(X_{t}-A_{t}\right) \mid F_{S}\right] .
\end{aligned}
$$

If we multiply by $\left(\left(X_{s}-A_{s}\right) / X_{s}\right)$, we can obtain the desired supermartingale.

\section{Conclusion}

In this paper, we use ordered Banach algebra and the algebra cone to define the deflator random variable and confirm that the $s$-price system $(C, \pi)$ is no locally free lunch and equivalent to $\widetilde{C} \cap X_{+} \neq \phi$, where $\widetilde{C}$ is the set of all limit points of convergence net in $C$ and the s-price system $(C, \pi)$ is s-viable if and only if a subcharacter functional $\left.\psi\right|_{C} \leq \pi$ exists. We validate that the s-price system $(C, \pi)$ is no-s-arbitrage if and only if a deflator process exists and confirm that the trading strategy $H$ is self-financing with respect to the deflator process if and only if $H$ is self-financing with respect to $S(t)$. No locally free lunch is a necessary condition to materialize a one to-one correspondence between the equivalent martingale measure $Q$ for the deflator process and the subcharacter $\varphi \in \Gamma$ such that $\left.\varphi\right|_{C}=\pi$. The uniformly integrable deflator process is also an important condition for generating an equivalent locally martingale measure and to prove that if $X$ is a $\Re^{d+1}$-valued semi martingale with nonnegative components defined on the filtered probability space $\left(\Omega, F,\left\{F_{t}\right\}_{t \in I}\right.$, and $\left.P\right)$, we can construct an equivalent local martingale measure for the deflator process.

\section{Data Availability}

The data used to support the findings of this study are available from the corresponding author upon request.

\section{Conflicts of Interest}

The authors declare that they have no conflicts of interest.

\section{References}

[1] E. Czkwanianc and A. Pazkiewicz, On Martingale Measures for Stochastic Processes with Discrete Time, Probability and Potential, Oxford University Press, New York, NY, USA, 1978.

[2] J. M. Harrison and D. M. Kreps, "Martingales and arbitrage in multiperiod securities markets," Journal of Economic Theory, vol. 20, no. 3, pp. 381-408, 1979.

[3] F. Delbaen, "Representing martingale measures when asset prices are continuous and bounded," Mathematical Finance, vol. 2, no. 2, pp. 107-130, 1992.

[4] D. M. Kerps, "Arbitrage and equilibrium in economics wit infinitely many commodities," Journal of Mathematical Economics, vol. 8, pp. 15-35, 1981.

[5] R. C. Dalang, A. Morton, and W. Willinger, "Equivalent martingale measures and no-arbitrage in stochastic securities markets models," Stochastics and Stochastic Reports, vol. 29, no. 2, pp. 185-201, 1990.

[6] J. M. Harrison and D. M. Kreps, "Martingales and stochastic inferals in the theory continues trading," Stochastic Processes and Applications, vol. 11, pp. 215-260, 1981.

[7] K. Back and S. R. Pliska, "On the fundamental theorem of asset pricing with an infinite state space," Journal of Mathematical Economics, vol. 20, no. 1, pp. 1-18, 1991.

[8] W. Schachermayer, No Arbitrage: On the Work of David Kreps, Springer, Berlin, Germany, 1992.

[9] W. Schachermayer, "A Hilbert space proof of the fundamental theorem of asset pricing in finite discrete time," Insurance: Mathematics and Economics, vol. 11, no. 4, pp. 249-257, 1992.

[10] F. Delbaen and W. Schachermayer, "A General version of the fundamental theorem of asset pricing," Mathematische Annalen, vol. 300, no. 1, pp. 463-520, 1994.

[11] F. Delbaen and W. Schachermayer, "The fundamental theorem of asset pricing for unbounded stochastic processes," Mathematische Annalen, vol. 312, no. 2, pp. 215-250, 1998.

[12] S. A. Clark, "The valuation problem in arbitrage price theory," Journal of Mathematical Economics, vol. 22, no. 5, pp. 463478, 1993.

[13] W. Schachermayer, "Martingale measures for discrete-time processes with infinite horizon," Mathematical Finance, vol. 4, no. 1, pp. 25-55, 1994.

[14] Y. Kabanov and C. Stricker, On Equivalent Martingale Measures with Bounded Densities, Springer-Verlag, Berlin, Germany, 2001.

[15] Y. Kabanov and M. Safarian, Markets with Transaction Costs, Springer-Verlag, Berlin, Germany, 2009.

[16] Z. Chen, "Viable costs and equilibrium price in frictional securities markets," Annals of Economics and Finances, vol. 2, pp. 297-323, 2001.

[17] N. Gaussel, The Martingale Property of Prices when Arbitrage Opportunities Exist, University of Paris I, Paris, France, 2002.

[18] Y. Kabanov, "In discrete time a local martingale is a martingale under an equivalent probability measure," Finance and Stochastics, vol. 12, no. 3, pp. 293-297, 2008. 
[19] B. Y. Hussein, "On equivalent (super/sub) martingale measure," Journal of Babylon University, vol. 19, no. 3, 2011.

[20] B. Y. Hussein, "On equivalent martingale measures on $L^{p_{-}}$ space," Al- Qadisiyah Journal, vol. 2, no. 1, 2010.

[21] B. Y. Hussein and S. Fahim, "Real characterization on order Banach algebra," Journal of Advance in Mathematics, vol. 12, no. 9, 2016.

[22] V. Prkaj and J. Ruf, Local Martingale in Discrete Time, Cornell University, New York, NY, USA, 2018.

[23] J. B. Conway, A Course in Functional Analysis, Springer, New York, NY, USA, 1990.

[24] R. B. Ash, Real Analysis and Probability, Academic Press, NewYork, NY, USA, 1992. 